\title{
The Impact of Type 2 Diabetes on Circulating Adipokines in Patients with Metabolic Syndrome
}

\author{
Karin Schindler ${ }^{a} \quad G^{2}$ reisa Vila ${ }^{a}$ Friedrich Hoppichler ${ }^{b, c}$ \\ Monika Lechleitner ${ }^{c, d}$ Anton Luger $^{a}$ Christian Anderwald ${ }^{a}$ \\ Jürgen Hoefler ${ }^{b, c}$ Goran Tomasec ${ }^{b, c}$ Alexandra Kautzky-Willer ${ }^{a}$ \\ Bernhard Ludvik ${ }^{a}$ \\ a Division of Endocrinology and Metabolism, Department of Internal Medicine III, Medical \\ University Vienna, Vienna, ${ }^{\text {b}}$ Internal Medicine, Hospital Barmherzige Brüder, ${ }^{c}$ SIPCAN save \\ your life, Salzburg, dinternal Medicine, Hospital Hochzirl, Hochzirl, Austria
}

\author{
Key Words \\ Adipokine $\cdot$ Metabolic syndrome $\cdot$ Diabetes $\cdot$ GDF15 - Visfatin
}

\begin{abstract}
Aim: The aim of the study was to investigate, whether type 2 diabetes independently influences adipokines and inflammatory markers in patients with metabolic syndrome. Methods: 36 patients with metabolic syndrome without type 2 diabetes and 39 patients with metabolic syndrome with type 2 diabetes, carefully matched for age, sex, and BMI, were investigated. Primary outcome measures were circulating adipokines and inflammatory markers (adiponectin, leptin, visfatin, vaspin, resistin, TNF- $\alpha$, IL-6, monocyte chemoattractant protein-1 (MCP-1), retinol binding protein 4 (RBP-4), growth differentiation factor-15 (GDF-15)). In addition, we determined parameters of glucose and lipid metabolism. Results: Patients with metabolic syndrome and type 2 diabetes had significantly lower levels of plasma total cholesterol, lowdensity lipoprotein cholesterol and triglycerides $(p<0.05)$. They displayed higher GDF-15 concentrations $(1,113 \pm 135 \mathrm{vs} .656 \pm 63 \mathrm{pg} / \mathrm{ml}, \mathrm{p}=0.005)$ and lower visfatin concentrations ( $3.7 \pm 0.3$ vs. $4.8 \pm 0.2 \mathrm{ng} / \mathrm{ml}, \mathrm{p}=0.009$ ). There were no differences in other adipokines and inflammatory markers between both groups. Conclusions: In patients with metabolic syndrome, type 2 diabetes leads to decreased visfatin and increased GDF-15 serum levels when compared to carefully matched non-diabetic subjects. Whether the increase in GDF-15 is an indicator or a causal factor for the increased cardiovascular risk in diabetic subjects remains to be investigated in further studies.


Schindler et al.: The Impact of Type 2 Diabetes on Circulating Adipokines in Patients with Metabolic Syndrome

\section{Introduction}

Abdominal obesity, insulin resistance, dyslipidemia, and hypertension constitute the hallmarks of the metabolic syndrome [1]. Patients with metabolic syndrome present a higher incidence of type 2 diabetes, which additionally increases their cardiovascular risk and overall mortality [2]. Regardless of the presence of type 2 diabetes, patients with metabolic syndrome exhibit an increased mass of visceral adipose tissue which results in altered adipokine release. There is evidence that this changed pattern of circulating adipokines contributes to insulin resistance and that cytokines such as IL- 6 and TNF- $\alpha$, which are released from adipose tissue macrophages, may act as pro-inflammatory signals [3, 4].

Several clinical studies have evaluated the relationship between circulating adipokines and insulin secretion and signaling [4-6]. Some adipokines, such as visfatin and leptin, are found to modulate beta-cell function [7,8]. Adiponectin, on the other hand, influences insulin sensitivity, and low circulating concentrations of adiponectin are associated with insulin resistance and the incidence of diabetes [9]. Recently, adipocytes were found to secrete also the growth differentiation factor-15 (GDF-15) [10]. GDF-15 was previously known as a product of stress-activated macrophages, endothelial cells, and cardiomyocytes [11, 12]. GDF-15 predicts cardiovascular events and is found to be increased in obesity and even further in obese patients with accompanying type 2 diabetes $[12,13]$. Nevertheless, there are no studies comparing circulating GDF-15 concentrations in carefully matched metabolic syndrome patients with and without type 2 diabetes.

In this study we aimed to address this question measuring circulating concentrations of adipokines in patients with metabolic syndrome with and without diabetes. Given the important impact of age, gender, and BMI on adipokine patterns, both groups were carefully matched for these variables.

\section{Material and Methods}

\section{Study Population}

Patients attending the obesity outpatient clinic of the Division of Endocrinology and Metabolism at the Medical University of Vienna were recruited in an ongoing, prospective cohort study approved by the Institutional Review Board. During the routine examination in the outpatients department, participants underwent a thorough medical examination including the measurements of weight (to the nearest $100 \mathrm{~g}$ ), height, waist and hip circumference (to the nearest centimeter), and blood pressure (using a sphygmomanometer and cuff appropriate for the arm circumference). Mean arterial pressure (MAP) was calculated as $(2 \times$ diastolic blood pressure + systolic blood pressure)/ 3 as previously described [14]. Glucose, triglycerides, total cholesterol, low-density lipoprotein (LDL) cholesterol, high-density lipoprotein (HDL) cholesterol, creatinine, albumine, insulin, C-peptide, TSH, and HbA1c were measured using routine tests in a certified laboratory (www.kimcl.at). Additional blood samples were collected for the measurement of adipokines, centrifuged at 3,500 rpm for $10 \mathrm{~min}$ and then immediately frozen at $-20^{\circ} \mathrm{C}$ ( www.biobank.at). All patients with previously undiagnosed diabetes underwent an oral glucose tolerance test (OGTT) using 75 g glucose.

For the present study, we selected from this cohort two groups including 36 patients with metabolic syndrome without type 2 diabetes and 39 patients with metabolic syndrome and type 2 diabetes carefully matched for age, gender, and BMI. Metabolic syndrome was defined using the NCEP-ATP3 criteria [15]. Diabetes mellitus was diagnosed using the American Diabetes Association criteria [16]. Patients receiving insulin were excluded, as insulin directly impacts the production of several adipokines. 37 out of 39 patients having diabetes were on oral antidiabetic treatment. Other exclusion criteria were the presence of a positive medical history for coronary heart disease, heart failure, peripheral artery disease, stroke, malignancy, and chronic liver or renal disease. Clinical, biochemical and metabolic characteristics of the study participants are presented in table $1.50 \%$ of the patients with metabolic syndrome without diabetes and $53 \%$ of the patients with metabolic syndrome and diabetes were taking lipid-lowering drugs. 
In addition, a third age- and gender matched group of 33 healthy subjects (age $51 \pm 1.2$ years; 14 males, 19 females) with normal BMI (mean $23.5 \mathrm{~kg} / \mathrm{m}^{2}$, range $18.7-25 \mathrm{~kg} / \mathrm{m}^{2}$ ), normal OGTT, and no present acute or chronic illness was included in the study. In this group, baseline blood samples were taken for the measurement of circulating GDF-15 concentrations.

Assays

Concentrations of leptin and adiponectin were analyzed using RIA kits from Linco Research (St. Charles, MO, USA) both with inter- and intra-assay coefficients of variation 5-8\%. Vaspin was determined using ELISA kits from AdipoGen (Seoul, South Korea) with intra- and inter-assay coefficients of variation being $2.76 \%$ and $3.27 \%$, respectively. Visfatin was measured using an EIA from Phoenix Pharmaceuticals (Karlsruhe, Germany) with inter- and intra-assay coefficients of variation $<15 \%$ and $<10 \%$, respectively.

Concentrations of retinol binding protein 4 (RBP-4), resistin, GDF-15 and monocyte chemoattractant protein-1 (MCP-1) were determined using specific ELISA kits from R\&D (Minneapolis, MN, USA). Inter- and intra-assay coefficients of variation were $<8.6 \%$ and $<8.1 \%$ for RBP- $4,<9.2 \%$ and $<5 \%$ for resistin, $<6 \%$ and $<2.8 \%$ for GDF-15, and $<5.9 \%$ and $<7.8 \%$ for MCP-1, respectively. Concentrations of TNF- $\alpha$ and IL- 6 were measured using high-sensitivity ELISAs from R\&D, with inter- and intra-assay coefficients of variation of $<10.6 \%$ and $<8.5 \%$ for TNF- $\alpha$ and $<9.6 \%$ and $<7.8 \%$ for IL-6, respectively. All samples were analyzed in duplicates and using kits of the same batch number.

\section{Statistical Analysis}

Data are expressed as mean \pm SE. Distribution was tested for normality using histograms. Differences between the groups were tested using the Mann-Whitney U test for non-parametric data and the Bonferroni-Holm corrected two-sided independent-samples t test for parametric data. The statistical software package SPSS release 15.0.1 (SPSS, Inc., Chicago, IL, USA) was used. P values less than 0.05 were considered statistically significant.

\section{Results}

Patients with type 2 diabetes displayed higher glucose $(\mathrm{p}=0.004)$ and HbA1c values ( $7.7 \pm 0.3 \%$ vs. $5.8 \pm 0.1 \%, p<0.001)$, but reduced total cholesterol $(217 \pm 8$ vs. $248 \pm 8$ $\mathrm{mg} / \mathrm{dl}, \mathrm{p}=0.01)$ and LDL cholesterol levels $(129 \pm 7$ vs. $151 \pm 6 \mathrm{mg} / \mathrm{dl}, \mathrm{p}=0.03)$ when compared to patients without diabetes (table 1).

There were no differences in leptin, adiponectin, RBP-4, vaspin, resistin, TNF- $\alpha$, IL-6 and MCP-1 between the groups (table 2). GDF-15 concentrations were increased in patients with metabolic syndrome in the absence and presence of diabetes when compared to ageand gender-matched healthy control subjects $(\mathrm{p}=0.002$ and $\mathrm{p}<0.001$, respectively; fig. 1$)$. In patients with metabolic syndrome, the presence of diabetes was associated with a $70 \%$ increase in plasma GDF-15 concentrations ( $p=0.005$; fig. 1 ) and with a $23 \%$ decrease in circulating visfatin ( $\mathrm{p}=0.009$; fig. 2 ). The association of low visfatin levels with the presence of diabetes remained significant also after adjusting for the concomitant intake of lipidlowering drugs $(\mathrm{p}=0.011)$.

\section{Discussion}

Patients with metabolic syndrome are at higher risk for developing diabetes [2]. When carefully matched for the features of the metabolic syndrome, patients with type 2 diabetes exhibit decreased circulating visfatin and increased GDF-15 concentrations, but no differences in other measured adipokines. Increased GDF-15 levels were previously observed in obese patients with diabetes, who were older than the control subjects [13]. As age counts among the main determinants of GDF-15 levels it was till now not clear whether the found 
Table 1. Characteristics of the study participants
DOI: 10.1159/000338729

Published online: April 21, 2012

(C) 2012 S. Karger GmbH, Freiburg www.karger.com/ofa

Schindler et al.: The Impact of Type 2 Diabetes on Circulating Adipokines in Patients with Metabolic Syndrome

\begin{tabular}{llll}
\hline & \multicolumn{2}{l}{ Metabolic syndrome } & p value \\
\cline { 2 - 3 } & $\begin{array}{l}\text { without diabetes } \\
(\mathrm{n}=36)\end{array}$ & $\begin{array}{l}\text { with diabetes } \\
\text { (n=39) }\end{array}$ & \\
\hline Sex, male/female & $13 / 23$ & $15 / 24$ & n.s. \\
Age, years & $51.2 \pm 1.9$ & $54 \pm 1.8$ & n.s. \\
BMI, kg/m ${ }^{2}$ & $33.4 \pm 1.2$ & $34.7 \pm 1$ & n.s. \\
MAP, mm Hg & $106 \pm 2$ & $111 \pm 2$ & n.s. \\
Fasting glucose, mg/dl & $102 \pm 4$ & $129 \pm 8$ & 0.004 \\
Fasting insulin, $\mu \mathrm{U} / \mathrm{ml}$ & $28 \pm 3.2$ & $24.4 \pm 2.6$ & n.s. \\
HOMA-IR & $7 \pm 0.8$ & $7 \pm 0.9$ & n.s. \\
HbA1c, \% & $5.8 \pm 0.1$ & $7.7 \pm 0.3$ & $<0.001$ \\
Triglycerides, mg/ml & $306 \pm 34$ & $245 \pm 37$ & n.s. \\
Total cholesterol, mg/dl & $248 \pm 8$ & $217 \pm 8$ & 0.01 \\
LDL-cholesterol, mg/dl & $151 \pm 6$ & $129 \pm 7$ & 0.03 \\
HDL-cholesterol, mg/dl & $47 \pm 2$ & $46 \pm 2$ & n.s. \\
Creatinine, mg/dl & $0.95 \pm 0.02$ & $1.16 \pm 0.2$ & n.s. \\
\hline
\end{tabular}

aData are presented as mean $\pm \mathrm{SE}$.

MAP = Mean arterial pressure; HOMA-IR = homeostatic model assessment insulin resistance index; LDL = low-density lipoprotein; HDL = high-density lipoprotein; n.s. = not significant.
Table 2. Adipokine and adipocytokine levels in patients with metabolic syndrome in the presence and in the absence of diabetes

\begin{tabular}{llll}
\hline & \multicolumn{2}{l}{ Metabolic syndrome } & p value \\
\cline { 2 - 3 } Parameter & $\begin{array}{l}\text { without diabetes } \\
(\mathrm{n}=36)\end{array}$ & $\begin{array}{l}\text { with diabetes } \\
(\mathrm{n}=39)\end{array}$ \\
\hline Leptin, ng/ml & $24.1 \pm 2.6$ & $17.7 \pm 1.9$ & n.s. \\
Adiponectin, $\mu \mathrm{g} / \mathrm{ml}$ & $8.2 \pm 0.5$ & $10.2 \pm 1.3$ & n.s. \\
RBP-4, $\mu \mathrm{g} / \mathrm{ml}$ & $40 \pm 1.7$ & $39 \pm 1.7$ & n.s. \\
Vaspin, ng/ml & $0.76 \pm 0.1$ & $0.63 \pm 0.1$ & n.s. \\
Resistin, ng/ml & $12.8 \pm 0.8$ & $12.7 \pm 0.8$ & n.s. \\
Visfatin, ng/ml & $4.8 \pm 0.2$ & $3.7 \pm 0.3$ & 0.009 \\
GDF-15, pg/ml & $656 \pm 63$ & $1,113 \pm 135$ & 0.005 \\
TNF- $\alpha, \mathrm{pg} / \mathrm{ml}$ & $7.3 \pm 1.9$ & $5.8 \pm 1.4$ & n.s. \\
IL-6, pg/ml & $3.3 \pm 0.6$ & $2.7 \pm 0.4$ & n.s. \\
MCP-1, pg/ml & $397 \pm 22$ & $442 \pm 19$ & n.s. \\
\hline
\end{tabular}

aData are presented as mean $\pm \mathrm{SE}$.

RBP-4 = Retinol binding protein 4; GDF-15 = growth differentiation factor 15; TNF- $\alpha=$ tumor necrosis factor alpha; IL-6 = interleukin 6 ; MCP-1 = monocyte chemoattractant protein-1; n.s. = not significant. 
Fig. 1. Circulating concentrations of GDF-15 in patients with metabolicsyndromeand diabetes (MS-DM), in patients with metabolic syndrome without diabetes (MS) and healthy controls.

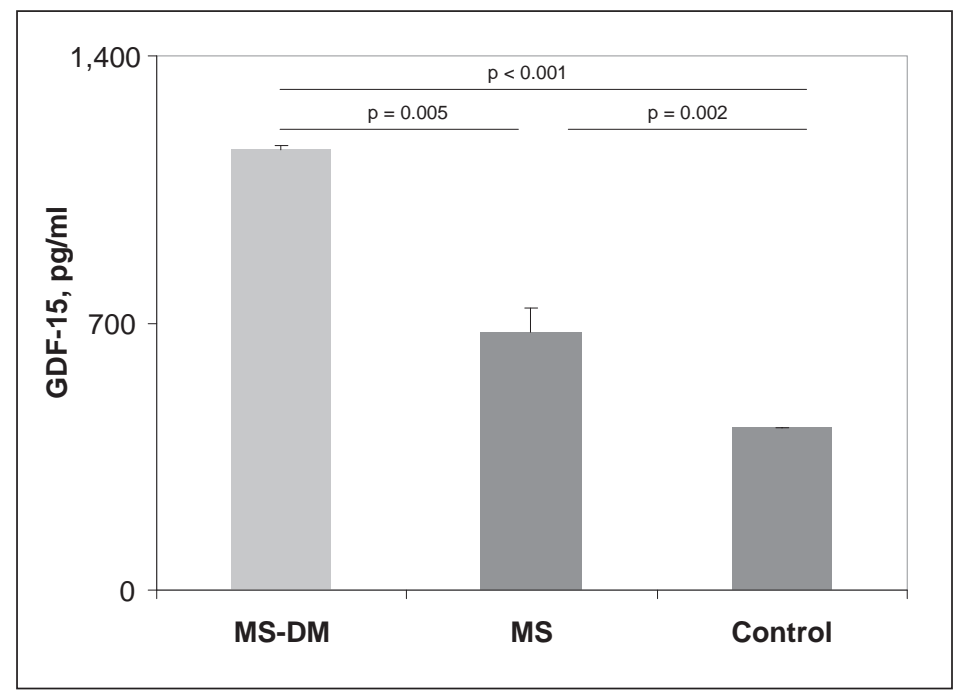

Fig. 2. Circulating concentrations of visfatin in patients with metabolic syndrome and diabetes (MS-DM) and in patients with metabolic syndrome without diabetes (MS).

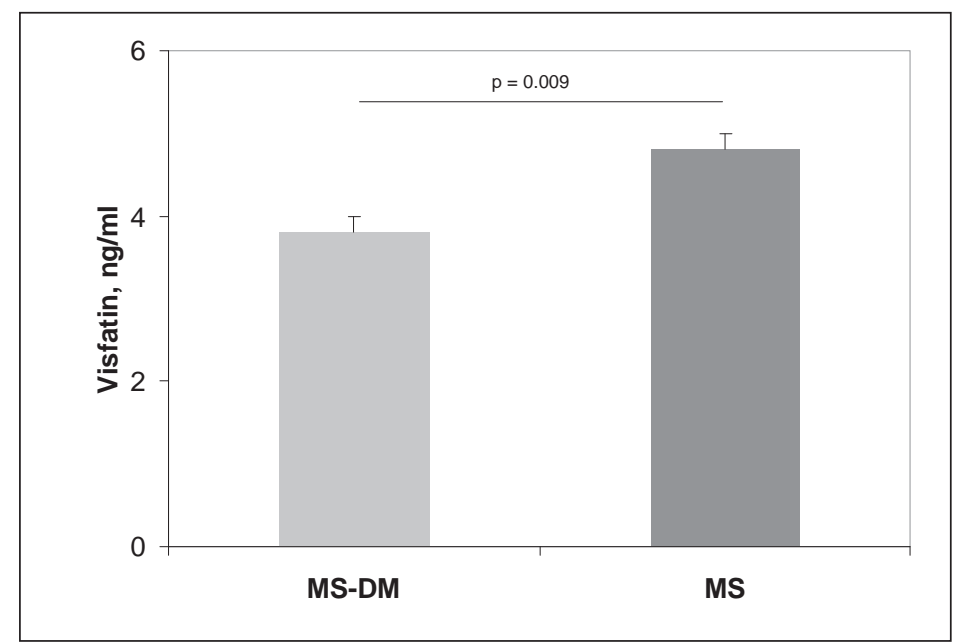

increases in GDF-15 concentrations were directly depending on the presence of diabetes or indirectly depending on the fact that the incidence of diabetes increases with age. The present study is the first study to show a significant increase in circulating GDF-15 in diabetic patients as compared to a carefully matched control group regarding the features of the metabolic syndrome. The pathophysiological mechanisms underlying the relationship of substantially higher GDF-15 levels and the presence of diabetes are still unknown $[13,17]$. Nevertheless, the fact that GDF-15 has recently emerged as an adverse cardiovascular prognostic marker emphasizes the increased cardiovascular risk in patients with metabolic syndrome accompanied by diabetes. In this regard, it is of interest that elevated levels of GDF-15 have been linked to an increased risk of cardiovascular events and mortality in type 1 diabetic patients with nephropathy [18]. To date, there are no data on metabolic pathways controlling GDF-15. Therefore, it is not known whether the increase in GDF-15 concentrations in diabetic subjects is due to the increase in glucose levels or to the unfavorable profile of concomitant cardiovascular risk factors which accompany type 2 diabetes. 
The presence of type 2 diabetes affected only plasma visfatin, leading to reduced visfatin concentrations in the absence of changes in other adipokines. The relationship between visfatin and insulin has been investigated in many studies [5]. It is interesting to note that visfatin was initially found to be increased in patients with type 2 diabetes [19]. Later studies found contradicting results $[20,21]$. In this study we demonstrate that patients with metabolic syndrome display lower visfatin plasma levels in the presence of diabetes. Visfatin stimulates insulin secretion from pancreatic islets [8]; therefore, lower visfatin levels might contribute to the development of diabetes in the context of metabolic syndrome. Thus, the discrepant results from studies on plasma visfatin levels in patients with diabetes mellitus might be attributed to differences in the study populations and may be strongly affected by the presence or absence of accompanying risk factors and the metabolic syndrome.

It is interesting to note that the complication of metabolic syndrome by diabetes is not associated with differences in leptin, adiponectin, RBP-4, vaspin, resistin as well as in the adipocytokines TNF- $\alpha$, IL- 6 and MCP-1. Although many of these parameters are found to influence insulin secretion and signaling $[3,4]$, they do not seem to be related to the development of diabetes in the clinical context of metabolic syndrome.

In summary, the presence of diabetes in patients with metabolic syndrome is associated with increased levels of GDF-15 and reduced levels of the insulinotropic adipokine visfatin. Whether these differences are markers of or causally involved in the increased cardiovascular complications of patients with type 2 diabetic remains to be determined.

\section{Acknowledgements}

The study was designed by BL, FH, ML, and AL. Data were acquired by KS, BL, and GV. KS and GV analyzed the data. $\mathrm{BL}, \mathrm{AL}, \mathrm{ML}, \mathrm{FH}, \mathrm{GV}$, and $\mathrm{KS}$ were responsible for interpretation of the data. $\mathrm{KS}, \mathrm{GV}, \mathrm{FH}, \mathrm{ML}, \mathrm{AL}, \mathrm{JH}$, GT, AK, and BL wrote and reviewed the manuscript.

This study was supported by Nestlé / Nestec Ltd. and Propter Homines Foundation, which is gratefully acknowledged.

\section{Disclosure Statement}

The authors declare that there is no duality of interest associated with this manuscript.

\section{References}

1 Alberti KG, Eckel RH, Grundy SM, Zimmet PZ, Cleeman JI, Donato KA, Fruchart JC, James WP, Loria CM, Smith SC Jr: Harmonizing the metabolic syndrome: a joint interim statement of the International Diabetes Federation Task Force on Epidemiology and Prevention; National Heart, Lung, and Blood Institute; American Heart Association; World Heart Federation; International Atherosclerosis Society; and International Association for the Study of Obesity. Circulation 2009;120:1640-1645.

2 Meigs JB: Epidemiology of type 2 diabetes and cardiovascular disease: translation from population to prevention: the Kelly West award lecture 2009. Diabetes Care 2010;33:1865-1871.

- 3 Hotamisligil GS: Inflammation and metabolic disorders. Nature 2006;444:860-867.

- 4 Rasouli N, Kern PA: Adipocytokines and the metabolic complications of obesity. J Clin Endocrinol Metab 2008;93(11 suppl 1):S64-73.

- 5 Haider DG, Schaller G, Kapiotis S, Maier C, Luger A, Wolzt M: The release of the adipocytokine visfatin is regulated by glucose and insulin. Diabetologia 2006;49:1909-1914.

- 6 Promintzer M, Krebs M, Todoric J, Luger A, Bischof MG, Nowotny P, Wagner O, Esterbauer H, Anderwald C: Insulin resistance is unrelated to circulating retinol binding protein and protein $\mathrm{C}$ inhibitor. J Clin Endocrinol Metab 2007;92:4306-4312. 
7 Maedler K, Schulthess FT, Bielman C, Berney T, Bonny C, Prentki M, Donath MY, Roduit R: Glucose and leptin induce apoptosis in human beta-cells and impair glucose-stimulated insulin secretion through activation of c-Jun N-terminal kinases. FASEB J 2008;22:1905-1913.

8 Revollo JR, Korner A, Mills KF, Satoh A, Wang T, Garten A, Dasgupta B, Sasaki Y, Wolberger C, Townsend RR, Milbrandt J, Kiess W, Imai S: Nampt/PBEF/visfatin regulates insulin secretion in beta cells as a systemic NAD biosynthetic enzyme. Cell Metab 2007;6:363-375.

9 Fagerberg B, Kellis D, Bergstrom G, Behre CJ: Adiponectin in relation to insulin sensitivity and insulin secretion in the development of type 2 diabetes: A prospective study in 64-year-old women. J Intern Med 2011;269:636-643.

10 Ding Q, Mracek T, Gonzalez-Muniesa P, Kos K, Wilding J, Trayhurn P, Bing C: Identification of macrophage inhibitory cytokine-1 in adipose tissue and its secretion as an adipokine by human adipocytes. Endocrinology 2009;150:1688-1696.

-11 Bootcov MR, Bauskin AR, Valenzuela SM, Moore AG, Bansal M, He XY, Zhang HP, Donnellan M, Mahler S, Pryor K, Walsh BJ, Nicholson RC, Fairlie WD, Por SB, Robbins JM, Breit SN: MIC-1, a novel macrophage inhibitory cytokine, is a divergent member of the TGF-beta superfamily. Proc Natl Acad Sci U S A 1997;94:1151411519.

-12 Taddei S, Virdis A: Growth differentiation factor-15 and cardiovascular dysfunction and disease: malefactor or innocent bystander? Eur Heart J 2010;31:1168-1171.

13 Vila G, Riedl M, Anderwald C, Resl M, Handisurya A, Clodi M, Prager G, Ludvik B, Krebs M, Luger A: The relationship between insulin resistance and the cardiovascular biomarker growth differentiation factor-15 in obese patients. Clin Chem 2011;57:309-316.

-14 Riedl M, Vila G, Maier C, Handisurya A, Shakeri-Manesch S, Prager G, Wagner O, Kautzky-Willer A, Ludvik B, Clodi M, Luger A: Plasma osteopontin increases after bariatric surgery and correlates with markers of bone turnover but not with insulin resistance. J Clin Endocrinol Metab 2008;93:2307-2312.

15 Grundy SM, Brewer HB Jr, Cleeman JI, Smith SC Jr, Lenfant C: Definition of metabolic syndrome: report of the National Heart, Lung, and Blood Institute/American Heart Association conference on scientific issues related to definition. Circulation 2004;109:433-438.

16 ADA: Diagnosis and classification of diabetes mellitus. Diabetes Care 2008;31(suppl 1):S55-60.

$\checkmark 17$ Lajer M, Jorsal A, Tarnow L, Parving HH, Rossing P: Plasma growth differentiation factor-15 independently predicts all-cause and cardiovascular mortality as well as deterioration of kidney function in type 1 diabetic patients with nephropathy. Diabetes Care 2010;33:1567-1572.

-18 Lind L, Wallentin L, Kempf T, Tapken H, Quint A, Lindahl B, Olofsson S, Venge P, Larsson A, Hulthe J, Elmgren A, Wollert KC: Growth-differentiation factor-15 is an independent marker of cardiovascular dysfunction and disease in the elderly: results from the Prospective Investigation of the Vasculature in Uppsala Seniors (PIVUS) Study. Eur Heart J 2009;30:2346-2353.

19 Chen MP, Chung FM, Chang DM, Tsai JC, Huang HF, Shin SJ, Lee YJ: Elevated plasma level of visfatin/pre-B cell colony-enhancing factor in patients with type 2 diabetes mellitus. J Clin Endocrinol Metab 2006;91: 295-299.

20 Haider DG, Schindler K, Bohdjalian A, Prager G, Luger A, Wolzt M, Ludvik B: Plasma adipocyte and epidermal fatty acid binding protein is reduced after weight loss in obesity. Diabetes Obes Metab 2007;9:761-763.

21 Li L, Yang G, Li Q, Tang Y, Yang M, Yang H, Li K: Changes and relations of circulating visfatin, apelin, and resistin levels in normal, impaired glucose tolerance, and type 2 diabetic subjects. Exp Clin Endocrinol Diabetes 2006;114:544-548. 